\title{
'Location is not enough!': an Empirical Study of Location-Awareness in Mobile Collaboration
}

\author{
Nicolas Nova, Fabien Girardin and Pierre Dillenbourg \\ CRAFT, School of Computer and Communication Sciences \\ Ecole Polytechnique Fédérale de Lausanne, CH-1015 Lausanne, Switzerland. \\ nicolas.nova@epfl.ch, fabien.girardin@epfl.ch, pierre.dillenbourg@epfl.ch
}

\begin{abstract}
:
There is an ever growing number of mobile learning applications based on location-awareness, However, there is still a lack of information concerning how it might impact socio-cognitive processes involved in collaboration. This is what the following empirical study aimed to address. We used a mobile and collaborative game, running on Tablet PCs, to test two conditions. On one hand, groups could see the positions of each member; while in the other location-awareness was not provided. All users could use the Tablet PC to communicate through annotations. We found no differences between the two conditions with regard to the task performance. Neither were there any differences in terms of cognitive workload. However, players without the location-awareness indications had a better representation of their partners' paths. They wrote more messages and better explicated their strategies. The paper concludes with remarks about how this can be taken into account by mobile learning practitioners.
\end{abstract}

\section{Introduction}

Mobile computing is a new trend in CSCL as stated by Roschelle [18]. There are more and more occurrences of research about using mobile devices such as PDA or mobile phones in this context. There is also a burst of location-based services, that is to say mobile applications that take advantage of location information. Learning is one of the domains in which these positioning technologies might have a great impact. A reasonable number of applications devoted to mobile learning have been developed in this area (see for instance [1] for a review). Casas et al. [8] provided a short summary of the existing technologies and their connections to learning in 2002. They envisioned few applications in which learning can benefit from positioning techniques like displaying social patterns of interaction, group configurations, or group migrations.

Overall there are two kinds of educational applications that can take advantage of positioning technologies, depending on how the location information is used. On the one hand, there are applications that track users' or objects' location and display them to their partners. This is meant to support collaboration among the group. On the other hand, knowing where the user is can lead the system to trigger specific events or to allow him/her to post messages bound to this specific location. This is meant to support information sharing and task-related activities.

Savannah [11] is an example of the former type of application. This game is an educational platform, in which children learn about the ecology of the African savannah. Groups of six children explore physical space equipped with GPS-enabled handhelds, discovering resources that lions need to survive. In [3], Benford et al. describe an ethnographical study of this location-based educational game. Researchers found that the inherent uncertainties caused by the positioning techniques lead to misunderstandings between the representation of participants' position and their real whereabouts. Positioning technology, GPS errors in this context, was then a factor of coordination difficulties.

Labscape [1] is an example of the second type of applications; it allows users to collect data when working out in the field. Location sensors allowed the users to visualize where readings have been taken. Conversely, in the Urban Pollution Monitoring Project [20], mobile carbon monoxide sensors combined with GPS allowed users to $\log$ 
exposure to pollution in urban environment. Data gathered outdoor has been used by groups of children to analyze the findings in classroom for understanding this pollution was affected by traffic. There were also various projects that employed positioning technologies in museums to guide visitors and help them exploring the place. Brown et al. [3] developed a mixed-reality system, which engages visitors in sharing their visit with others. A location-based system allows users to co-visit a museum, seeing each others when observing the exhibit. In this context, they found that locationawareness is a resource that can be used to (a) quickly move to their friends to look at the same thing (b) ground the conversation: by helping participants to quickly know what exhibit is being discussed.

Apart from those projects, research about location-awareness in collaborative learning appears to be more design-oriented than directed towards the understanding of the effects induced by this technological feature.

In this paper, our perspective is less focused on the learning applications per se, as in the aforementioned examples, than on the understanding of the socio-cognitive processes involved by this kind of environments and how they might be influenced by mobile environments and location-based applications. By sociocognitive, we refer to all the processes that make collaboration possible: communication, division of labor, inference about partners' activity or comprehension. We believe that a deeper understanding of how those processes are influenced by location-awareness is an important step towards the design of efficient mobile learning environment.

In line with our purposes, we set up a collaborative system, in the form of a mobile game, meant to elicit the collaborative behavior of users. We report on an experiment we ran to investigate how location-awareness impacts collaboration processes.

Prior to describing the game environment and the experiment we conducted, we first present our research scope. We then describe the methodology deployed, the game environment as well as the procedure and the participants. In the following sections, we report and discuss the results of the experiment. In the final section, we reflect on how this work can provide useful design guidelines for the use of location-awareness in mobile learning applications.

\section{Research question}

Our current research in the field of mobile technology/location-awareness with regard to its impacts on collaborative processes emerges from our studies about this very topic in virtual environments. Past research projects already addressed the importance of location-awareness in virtual space. It has been found that knowing the partners' whereabouts has positive impacts on:

- Coordination and division of labor [9].

- Construction of a shared understanding of the situation [9].

- Communication patterns [15] and referential communication [17].

- Task performance [16].

- Inferences about partners' intents and strategies [16].

A purpose of this research project is to investigate how these results hold in a mobile context. The point here is to investigate the role of location-awareness when the joint task is mobile, dynamic and embedded in an environment filled with people and artifacts. The reasons why we want to move from virtual to real space are simple. First, the role of space gets even more important and relevant as the interaction occurs among different places in real space, with various contexts. Second, mobile computing offers interesting perspectives for Computer Supported Collaborative Learning: it then proposes new situations where we need insights about how the collaborative processes are modified.

The main research question deals with the potential effects of a location-awareness tool, with regard to the socio-cognitive processed involved when people collaborate. We investigate whether location cues influence collaboration upon different variables: task performance, communication among peers as well as the task workload. We are also interested into how location information can modify the way peers build a model of their partners' activity.

This empirical study is an exploratory investigation that focuses on the use of locationawareness on collaboration in a spatial coordination task. The presence or absence of the location-awareness tool constitutes the experimental condition of the study. 


\section{Method}

We deployed a 'field experiment' approach [12]. Field experiments are quantitative experimental evaluations that are conducted out in the field, drawing from aspects of both qualitative field studies and lab experiments. They take advantage of both qualitative and quantitative studies. On the one hand it involves real users in an activity that occurs in the real world. On the other hand, we can control variables and have different experimental conditions.

\subsection{Environment}

CatchBob! is a mobile game in which groups of 3 teammates have to find a virtual object on our campus at EPFL in Lausanne. The dimensions of this 'field' are $850 \times 510$ meters. Completing the game requires the players to surround the object with a triangle formed by each participant's position in the real space. To reach this goal, they employ an application running on Tablet PCs as depicted on figure 1.

Another meaningful piece of information given by the software is an individual proximity sensor. It indicates whether the user is close or far from the object through the number of red bars displayed at the top of the interface. There is actually no object on the field; it only appears on the screen when the users are close to it. In addition, the tool also enables communication: players can synchronously annotate the map with the stylus. The annotations constantly fade out until they become completely invisible (after 4 minutes). When the players are close to the object, the triangle they have to form appears on the display; they then have to adjust it in a proper way.

In the experimental condition "without the location-awareness tool", players just see their own character as an avatar on the campus map. In the condition "with location awareness", player could update his or her partners' positions by clicking on a refresh button.

Even though finding the object could be carried out alone, the collaboration in this game lies in the fact that players have to coordinate to form the triangle surrounding the virtual object. It's not possible to finish the game without collaborating. We hence avoid the free rider effect.

All the players' interactions with the applications (positions, annotations, getting others' positions, connection loss) are logged on a server. We also developed a replay tool that allows to show the paths of each player. This application allows us to confront the players to a replay of the path they took during the game, as well as the actions they performed.

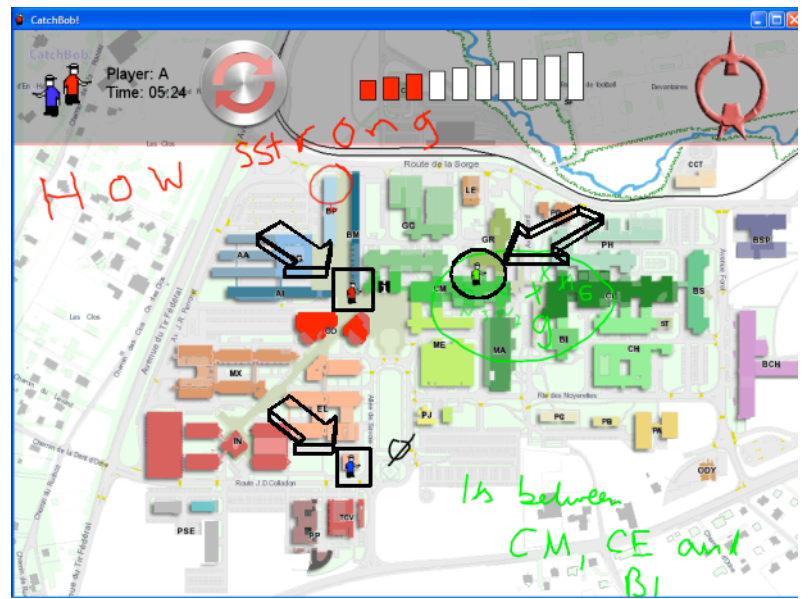

Figure 1. CatchBob! interface as seen by one player (surrounded by a circle). This snapshot depicts the interface with the location-awareness tool: avatars of other players in are displayed (surrounded by a square on this picture). In the condition without the location-awareness tool, the interface only displays the character's avatar (surrounded by a circle).

CatchBob! is fully written in Java (J2SE and J2EE). It uses a classical client-server communication architecture with SOAP as highestlevel protocol. The server hosts the game logic. Clients are in pull mode to synchronize and to constantly receive broadcasted messages. Tablet PCs self-determine their position. They interface with the Place Lab native libraries to retrieve the MAC address and the signal strength of nearby WiFi Access Points. With these data, a position is computed based on a propagation model and a basic centroid algorithm. CatchBob! can be run on many types of Tablet PCs, we conducted our experiments on the Compaq TC1000.

\subsection{Procedure and participants}

Sixty students of our University (age range: 1927; mean: 22.8) participated in this experiment. We had 10 groups of 3 persons in the condition "with awareness tool" and 10 groups in the condition "without awareness tool". All the group members knew each other and were familiar with the campus. Experiments lasted approximately one hour and were conducted in French. The experiments were run on our campus, one group at a time.

Participants were asked to find the virtual object and surround it with a triangle made by their 
position with one constraint in mind: they should take the shortest path to it. We told them that the goal was not to find the object in the smallest amount of time.

After presenting the game instructions at the lab, players were given 3 minutes to plan their strategy on a map. Players were then led to the common starting point at the centre of the campus. They had 30 minutes to complete the task. After completing the game (or playing 30 minutes), players returned to our lab and filled a post-game questionnaire during 10 minutes. This questionnaire provided participants with 3 maps of the campus on which they had to draw their path as well paths followed by the 2 partners. Players were also asked questions about how was the collaboration, if it was balanced or not, whether they had fun playing the game and if they understood their partners' intents during the joint task.

We controlled several variables like the number of participants among the group, the fact that they knew each other as well as the field, they have the same gear (a Tablet PC, no cell phone, no walkietalkie) and they have all the same starting point.

We used two different positions of "Bob". There is the same number of games with these 2 positions in each of the conditions. The distance between the starting point and Bob is the same in these 2 scenarios. We controlled that the position of 'Bob' had no effect on all the variables presented in the next section; which was not the case.

\subsection{Extracted data}

A wide set of measures can be extracted from CatchBob! They concern both performance and collaborative process indexes.

The performance index is the sum of the path length over all players in a group. We did not choose time as a performance variable since we did not want players to run on the campus with Tablet PC and because finding a proper path was better suited to the discussion of a relevant strategy.

In terms of the socio-cognitive processes involved, we measured three kinds of variables:

- The frequency and the content of annotations written on the Tablet PC reflect the communication among the group.

- The number of errors they made while drawing the path of their partners after the game is an indication of how each player modeled the activity of their partners.
- A NASA-TLX test [13] allowed us to grasp the subjective evaluation of the task workload, taking physical and cognitive load into account.

To compute the second 'process' index, we asked players to draw their path on a paper map as well as the paths of their partners, as described earlier. We could hence make comparisons between the path player $\mathrm{A}$ drawn about $\mathrm{B}$ or $\mathrm{C}$ to $\mathrm{B}$ or C's real paths. This comparison, measured by the number of mistakes, represents the quality of A's representation of B and C's behavior in space.

\section{Results}

\subsection{Statistical note}

We used non-parametric tests as Wilcoxon test when data were not distributed normally. When the distribution was normal, we used regular onewayANOVA analysis.

\subsection{Task Performance}

Since it was a collaborative game, we analyzed the task performance at the group level. The task performance corresponds to the path length of each group. As depicted on figure 2, groups in both conditions have a very close performance. A oneway-ANOVA test did not show significant differences.

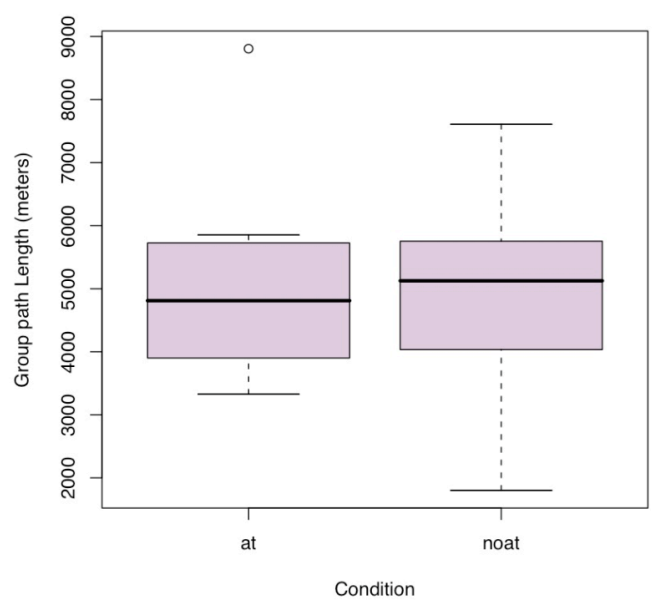

Figure 2. Path length of each group in the two conditions (at: with the location-awareness tool; noat: without the location-awareness tool) 


\subsection{Modeling the partners' paths}

As mentioned in the section about the experimental procedure, we measured the number of mistakes between the path player A drawn about $\mathrm{B}$ and $\mathrm{C}$ to $\mathrm{B}$ and $\mathrm{C}$ 's real paths. This represents the quality of A's representation of B and C's behavior in space. We did that for each player. This variable has been analyzed at the group level. As described by Kenny et al. [14], we checked the nonindependence of the results through the computation of intraclass correlation $(r=0.39)$, which is significant $(\mathrm{p}=0.01)$. That expresses the non-independence of the results among groups. It means that the number of errors made by the subjects is dependent on the number of errors did by the partners (e.g. if one player made a lot of errors about his/her path, the same goes for the partners). Then the unit of analysis is the group. Figure 3 shows the number of errors in each experimental condition.

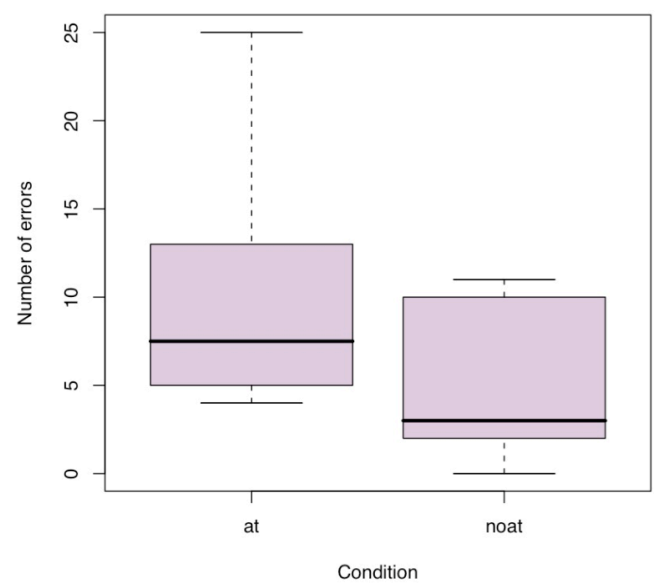

Figure 3. Number of errors made by each participant while drawing the path of their partners in the two conditions (at: with the locationawareness tool; noat: without the locationawareness tool).

Players without the location-awareness tool make two times less errors than those who had it. It shows that this difference is significant: $\mathrm{W}=81, \mathrm{p}$ $=0.02$.

\subsection{Map annotations}

The analysis of the messages sent by each individual on the map has also been investigated at two levels: frequency and content. This variable has been studied at the individual level since the intraclass correlation among the group is not significant $(r=-0.21 p=0.87)$. Figure 4 shows the frequency of messages sent by each player in both experimental conditions.

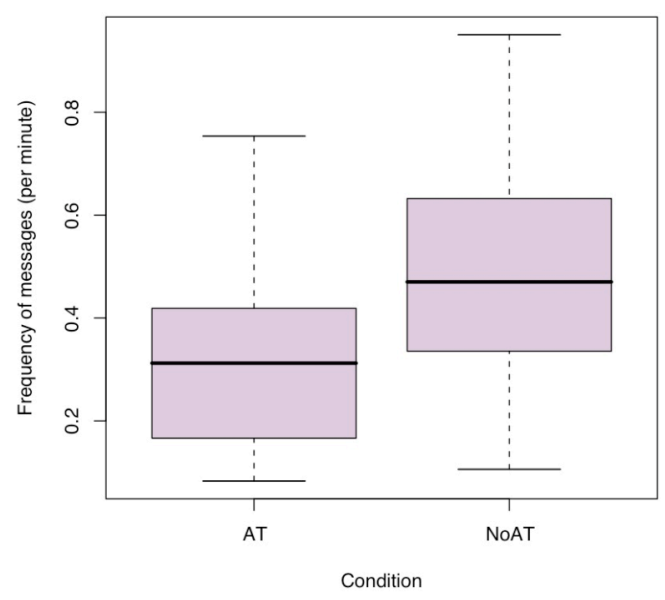

Figure 4. Frequency of messages sent by each participant in the two conditions (at: with the location-awareness tool; noat: without the locationawareness tool)

The frequency of messages is higher in the "without the location-awareness tool" condition. A Wilcoxon statistical test shows that this difference is significant: $\mathrm{W}=55.56 \mathrm{p}<0.01$.

We developed our own coding scheme to analyze map annotations. We coded the content of the messages (position, direction, strategy, proximity to the object, off-task, corrections) and their pragmatics (announcement, order, question, acknowledgement). In the condition without the awareness tool, the frequency of messages about position $(\mathrm{W}=203 \mathrm{p}<0.01)$ direction $(\mathrm{W}=292 \mathrm{p}=$ $0.01)$ and strategy $(\mathrm{W}=269 \mathrm{p}<0.01)$ is higher. In terms of pragmatics, players without the locationawareness tool sent more announcement $(\mathrm{W}=253$ $\mathrm{p}<0.01)$ and more questions $(\mathrm{W}=228.5 \mathrm{p}<0.01)$. There are no significant differences concerning the number of orders or acknowledgements.

In addition, there is a negative correlation between the frequency of messages about strategy and the number of errors made by the individual when drawing their partners' path: Pearson bivariate correlation $r=-.51$ (significant $\mathrm{p}<0.001$ ). In other words, strategy messages help to model the others' paths.

\subsection{Side results}

Using the NASA-TLX questionnaire, we had a quantitative evaluation of the perceived workload induced by this task. However, we did not find any 
differences between the two experimental conditions when looking at the index as a whole $(\mathrm{F}$ $=2.9, \mathrm{p}=0.09)$ or when isolating the cognitive workload $(\mathrm{W}=377$, not significant $)$ and the physical workload ( $\mathrm{W}=506$, not significant).

Finally, we analyzed the players' behavior when exploring the campus and two roles emerged: 'followers' and 'explorers'. The former refers to participants who stuck to the strategy they decided at the beginning, which is spreading over the campus and then rallying the one closer to the object as soon as possible. The latter role refers to players who kept exploring the campus even after being called by the one closer to the object. A chisquared test for independence reveals a difference between the repartition of 'followers' and 'explorer' in both conditions $(\mathrm{Chi}=6.35 \mathrm{p}=0.04)$. There were more 'explorers' in the condition without the location-awareness tool. This means that they reshape their strategy, optimizing it to better find the object. This was also reflected by the messages they sent since, as we saw in the previous section, there were more messages discussing the strategy.

\section{Discussion}

In sum, participants who were automatically aware of their partners' location did not perform the task better than other participants. In addition, people among groups without the location information did fewer errors when drawing the path of their partners after the game. These two results can be explained by the messages sent. First the amount of messages is more important in the group without the location-awareness tool. And when we look at the content, we see that players without the location-awareness tool sent more messages about position, direction or strategy. They also wrote more questions. It then appears that players without the awareness tool take better advantage of the annotation capabilities, using it to express their path and their strategy. We can then conclude that in the context of this experiment it was better to leave users without the location-awareness tool, with a broad channel of communication. They chose the information they perceived as relevant (position, direction and strategy) and sent them to their partners. In addition, players without the location-awareness tool were more willing to modify and reshape their strategy, especially through the use of map annotations that described how they would explore new zones of the campus. Finally, no differences have been found concerning the perceived workload due to the task (be it physical or cognitive).
These results confirm what has been found in another location-based game called Uncle Roy All Around You [3] in which Benford and his colleagues found that self-reported positioning could be reliable low-tech alternative to automated systems like GPS. Our findings goes further by proving that letting user declare themselves their position is better with regard to various processes like modeling the partners' intents or the (re)construction of the strategy.

Further analyses are directed towards the comprehension of specific events to investigate the role of location information on players' and group's behavior.

\section{Design Implications for Mobile Learning Environments}

In sum, we found that automatically giving the location-awareness information to participants is not always fruitful. In our case, it was better with regard to modeling the partners' intents to let users express what they estimated to be relevant through a broader channel of communication: the map annotations. The players with the awareness tool were able to annotate as well but did not use this opportunity. These results are close to what socioconstructivist theories [7] value in an educational context (i.e. elaborated explanations, selfregulation, strategies explicitations). Letting people build their own representation of the spatial information appears to be more efficient than broadcasting mere location information. To some extent, the fact that not giving location-awareness information was a way to support collaboration more effectively; since players communicated more and better explained their activity and intents, which led interestingly to reshape their strategy.

Another important benefit of letting the users express their position is to give them the control of privacy issues. They have indeed the choice to give or not to give information about their whereabouts, which is of tremendous importance to avoid the users' perception of privacy invasion.

Relying on the results gathered from this experiment, we have learned a number of design lessons. Concerning location-awareness to support collaboration, we differentiate three potential directions:

The first solution is to use the automatic positioning as a location-awareness tool, which is a trend followed by other scholars[4]. Nevertheless choosing this solution lead to different drawbacks ranging from low accuracy, privacy issues [5] and is sometimes not very efficient to support collaborative processes as found in our experiment. 
Based on these drawbacks this solution appears to have a strong influence on the socio-cognitive processes.

A second direction would be to rely on selfdeclared positioning. The main lesson we can draw here is that automatic location-awareness is not enough to promote collaboration. Even though the experiment did no reveal differences in task performance, self-declared positioning with written annotations appears to be better-suited and more relevant with regard to collaboration. In particular, there seems to be five advantages not to provide collaborative mobile users with a locationawareness tool:

- To facilitate the construction of a better mutual model: during this spatial coordination task we saw that players without the locationawareness tool built a more accurate representation of their partners' paths.

- To facilitate knowledge elicitation: without the automatic location-awareness, subjects were more articulate about their strategy. It seems that the tool created certain inertia among the group, with regard to communication. Participants who relied on the automatic positioning just wrote few messages, which lead them to be less explicit the situation and how they could deal with it.

- To ease conflict solving though a better explanation of what players want to do or to achieve in order to progress in the task completion. Being more verbose raised more conflicts, which is good for learning as stated by Doise and Mugny [10].

- Besides the absence of automatic positioning was not detrimental to task performance and it did not impact the cognitive workload of the task.

- In [3], authors also found that self-reported positioning provided excellent coverage and availability. Besides, in their case, users quickly learnt to use it.

As we saw in this paper, space and locationawareness is interesting but it should certainly not be limited to a simple broadcast of people's position. The results of this experiment show that it is better to let users control and express this information the way they want. Giving them the possibility to embed location cues with other kind of information like map annotations appeared to be a good solution to support collaborative processes like communication or strategy discussions. This is the reason why the title of this paper expresses the "Location is not enough" motto. Compared to automatic positioning in which location is just information, self-declared positioning is both an information and a communication act. Sending one's position to the partners is indeed at the same time a way to make manifest a fact that the player estimated as being relevant for the activity as explained by the 'relevance theory' of Sperber and Wilson [19]. Compared to the automatic positioning, the self-declaration of one's location is then bound to an intent: making clear and manifest that one is here and that it has sense for the joint activity.

Finally a third direction can be to benefit from automatic positioning solution to "augment" location-awareness. In this case, the spatial information automatically gathered can augment the information in the system while not being communicated just a simple position. We could imagine that the best mobile learning applications might then emerge from the synergy of automatic positioning and other interactions. For instance, in our case, the players' location can be used to position the map correctly or to position the messages sent by participants where they were.

\section{References}

[1] L. Arnstein, G. Borriello, S. Consolvo, C. Hung and J. Su. "Labscape: A Smart Environment for the Cell Biology Laboratory", IEEE Pervasive Computing Magazine, vol. 1, no. 3, July-September 2002, IEEE Computer Society, NY, NY

[2] S. Benford. Future Location-Based Experiences. JISC Tech Report (TSW0501), 2005, Retrieved from the Internet:

http://www.jisc.ac.uk/uploaded documents/jisctsw $05 \quad 0$ 1.pdf [last accessed 08/06/05].

[3] S. Benford, D. Rowland, M. Flintham, A. Drozd, R. Hull, J. Reid, J. Morrison, and K. Facer. "Life on the edge: supporting collaboration in location-based experiences". In Proceedings of the 2005 CHI Conference on Human Factors in Computing Systems, Portland, Oregon: ACM Press, 2005, pp. 721-730.

[4] S. Benford, W. Seagar, M. Flintham, R. Anastasi, D. Rowland, J. Humble, D. Stanton, J. Bowers, N. Tandavanitj, M. Adams, J.R. Farr, A. Oldroyd, and J. Sutton. "The error of our ways: The experience of selfreported position in a location-based game". In Proceedings of the 6th International Conference on Ubiquitous Computing. (UbiComp 2004), Nottingham., pp. 70-87,

[5] G. Borriello, M. Chalmers, A. LaMarca, and P. Nixon. "Delivering real-world ubiquitous location systems." Communications of the ACM, 48, 3, 2005, pp. 36-41. 
[6] B. Brown, I. McColl, M. Chalmers, A. Galani, C. Randel, and A. Steed. Lessons from the Lighthouse: Collaboration in a Shared Mixed Reality System. In Proceedings of the CHI 2003 Conference on Human Factors in Computing Systems, 2003, pp. 577--584. ACM Press.

[7] J.S. Brown, A. Collins and P. Duguid,. "Situated cognition and the culture of learning". Educational researcher, 18,1989 , pp. $32-42$.

[8] R. Casas, D. Cuartielles, J. Falco and L. Malmborg. "Positioning technologies in learning". In Proceedings of International Workshop on Wireless and Mobile Technologies in Education (WMTE 2002), Växjö, August 29-30, 2002, pp. 161-162.

[9] P. Dillenbourg and D. Traum. "Sharing solutions: persistence and grounding in multi-modal collaborative problem solving". To appear in Journal of Learning Sciences.

[10] W. Doise, and G. Mugny. The social development of the intellect. Pergamon Press, Oxford, 1984.

[11] K. Facer, R. Joiner, D. Stanton, J. Reid, R. Hull and D: Kirk. "Savannah: mobile gaming and learning?" Journal of Computer Assisted Learning, 20, pp. 399-409, 2004.

[12] J. Goodman, D. Brewster, and P. Gray. "Using Field Experiments to Evaluate Mobile Guides". In SchmidtBelz, B. and Cheverst, K., (eds.), Proceedings of HCI in Mobile Guides, workshop at Mobile HCI 2004, Glasgow, UK, Sep 2004.

[13] S.G. Hart and L.E. Staveland. "Development of NASATLX (Task Load Index): Results of Empirical and Theoretical Research". In Human Mental Workload, Hancock, P. and Meshkati, N. (eds.). North Holland B.V., Amsterdam, pp. 139-183, 1988.

[14] D.A. Kenny, D. Kashy and N. Bolger. Data analysis in social psychology. In D. Gilbert, S. Fiske, \& G. Lindzey (eds.) Handbook of Social Psychology, vol. 1, pp. 233-251. Boston: McGraw-Hill, 1998, pp. 233-265.

[15] L. Montandon . 'Etude des mécanismes de coordination spatiale dans un environnement virtuel de collaboration'. Mémoire de Diplôme d'Etudes Supérieures en Sciences et Technologies de l'Apprentissage. Non publié. TECFA, Faculté de Psychologie et des Sciences de l'Education, Université de Genève, 1996.

[16] N. Nova, T. Wehrle, J. Goslin, Y. Bourquin and P. Dillenbourg. "The Impacts of Awareness Tools on Mutual Modelling in a Collaborative Video-Game". in Proceedings of the 9th International Workshop on Groupware, (Autrans, France, September 2003), pp. 99108.
[17] D. Ott and P. Dillenbourg. "Using Proximity and View Awareness to Reduce Referential Ambiguity in a Shared 3D Virtual Environment". In Proceedings of CSCL 2002, (Boulder, CO, January 7-11, 2002), Lawrence Elbaum: NJ, pp. 603-604.

[18] J. Roschelle. "Unlocking the learning value of wireless mobile devices". Journal of Computer Assisted Learning, 19, 3, 2003, pp. 260-272.

[19] D. Sperber and D. Wilson. Relevance: Communication and cognition, Blackwell, Oxford, 1986.

[20] A. Steed, S. Spinello, B. Croxford, and C. Greenhalgh." e-Science in the Streets: Urban Pollution Monitoring", In Proceedings of the 2nd UK e-Science All Hands Meeting 2003. 